\title{
1 Evaluation of acute kidney injury in dogs with complicated or uncomplicated \\ Babesia rossi infection
}

\section{3}

12 luc.duchateau@ugent.be

13 d Department of Veterinary Science, University of Turin, Largo Braccini 2, 10095 Grugliasco, Torino, Italy

14 luca.aresu@unito.it

15 e Department of Pharmacology, Toxicology and Biochemistry, Faculty of Veterinary Medicine, Ghent University, Merelbeke, Belgium 16 evelyne.meyer@ugent.be

17

$18 *$ Corresponding author.

19 E-mail address: pieterdefauw@gmail.com (P. Defauw)

20 Present address: Lumbry Park Veterinary Specialists, Selborne Road, Alton, Hampshire GU34 3HL, United Kingdom.

21 This work was partially presented as a poster presentation at the ECVIM-ca congress in Rotterdam, The Netherlands, 6-8 September 2018.

25 Abbreviations:

AKI, acute kidney injury; ARDS, acute respiratory distress syndrome; ARF, acute renal failure; ATN, acute tubular necrosis; CKD, chronic kidney disease; CRP, C-reactive protein; ELISA, enzyme-linked immunosorbent assay; GFR, glomerular filtration rate; IgG, immunoglobulin G; IMHA, immune-mediated hemolytic anemia; IRIS, International Renal Interest Society; LM, light microscopy; NGAL, neutrophil gelatinase-associated lipocalin; OVAH, Onderstepoort Veterinary Academic Hospital; PAS, periodic acid-Schiff; RBP, retinol-binding protein; RLB, reverse line blot; sCr, serum creatinine; SDMA, symmetric dimethylarginine; TEM, transmission electron microscopy; uCr, urinary creatinine; UPC, urinary protein to creatinine ratio; USG, urine specific gravity; uOsmol, urine osmolality; UTI, urinary tract 32 infection 
Dogs with babesiosis can present with multiple complications, including acute kidney injury (AKI). The objective of this study was to characterize AKI in dogs with babesiosis caused by Babesia rossi at presentation and after treatment. Thirty-five client-owned dogs with B. rossi infection and 10 control dogs were included in this prospective observational study. Blood and urine were collected in Babesiainfected dogs at presentation $\left(T_{0}, n=35\right)$, after 24 hours $\left(T_{24 h}, n=11\right)$, and after 1 month $\left(T_{1 m}, n=9\right)$. The following urinary kidney injury biomarkers were assessed: urinary protein to creatinine ratio (UPC), urinary glomerular injury biomarkers (immunoglobulin G (uIgG) and C-reactive protein (uCRP)), and urinary tubular injury biomarkers (retinol-binding protein (uRBP) and neutrophil gelatinase-associated lipocalin (uNGAL)). Serum functional renal biomarkers were creatinine (sCr) and symmetric dimethylarginine (sSDMA). Post-mortem kidney biopsies were analyzed by light and transmission electron microscopy.

43 At $\mathrm{T}_{0}$, all kidney injury biomarkers were significantly higher in Babesia-infected dogs compared to healthy controls $(\mathrm{P}<0.001)$, while

44 functional renal biomarkers were not significantly different $(\mathrm{P}>0.05)$. At $\mathrm{T}_{24 \mathrm{~h}}$, all urinary tubular injury biomarkers and UPC decreased significantly $(\mathrm{P}<0.01)$, while glomerular injury biomarkers did not $(\mathrm{P}=0.084)$. At $\mathrm{T}_{1 \mathrm{~m}}$, all urinary kidney injury biomarkers decreased to values not significantly different from healthy controls $(\mathrm{P}>0.5)$. Significant changes in functional renal biomarkers were not seen after treatment $(\mathrm{P}>0.05)$. Dogs with complicated babesiosis had significantly higher glomerular injury biomarkers, UPC, and sSDMA compared to uncomplicated cases $(\mathrm{P}<0.05)$, while all tubular injury biomarkers and $\mathrm{sCr}$ were not significantly different $(\mathrm{P}>0.1)$. results. 


\section{Introduction}

Babesia rossi is an intra-erythrocytic protozoan, capable of causing life-threatening disease in infected dogs (Jacobson, 2006). Babesia species have different tick vectors, with B. rossi in sub-Saharan Africa being transmitted by Haemaphysalis elliptica (and presumably Haemaphysalis leachi) (Uilenberg et al., 1989; Apanaskevich et al., 2007; Penzhorn et al., 2017). Only the adult life stage of the tick is capable of transmitting B. rossi (Lewis et al., 1996). Because domestic dogs are not native to sub-Saharan Africa and B. rossi and its tick vector only occur in this region (Mitchell, 2015), it has been suggested that indigenous African canids are the reservoir hosts of B. rossi (Penzhorn, 2011). Evidence suggests that these indigenous African canids are able to cope with $B$. rossi infection and become asymptomatic carriers of this parasite (Penzhorn, 2011). Black-backed jackals were subsequently confirmed to be natural hosts of B. rossi in South Africa (Penzhorn et al., 2017). After introduction of domestic dogs into sub-Saharan Africa, Haemaphysalis ticks could not only infest their natural hosts, but also this newly introduced and highly susceptible domestic dog population, in whom the parasite causes disease because they would have had insufficient time to co-evolve with the B. rossi parasite (Penzhorn, 2011; Mitchell, 2015). In South Africa, severity of this disease ranges from mild to peracutely fatal (Jacobson and Clark, 1994). Babesia rossi-infected dogs can present with a variety of complications, including acute kidney injury (AKI), hypotension, hemoconcentration, immune-mediated hemolytic anemia (IMHA), hepatopathy, hypoglycemia, pancreatitis, cerebral babesiosis, and acute respiratory distress syndrome (ARDS) (Jacobson and Clark, 1994; Welzl et al., 2001; Keller et al., 2004; Jacobson, 2006; Defauw et al., 2012; Köster et al., 2015). There is accumulating evidence that an excessive pro-inflammatory host response is a major cause of these B. rossi-associated complications (Welzl et al., 2001; Köster et al., 2015; Goddard et al., 2016). Several studies documented that dogs infected with B. rossi often present with mild, non-azotemic manifestations of AKI, while overt acute renal failure (ARF) is less common (Jacobson and Clark, 1994; Lobetti and Jacobson, 2001; Welzl et al., 2001; Defauw et al., 2012). In a recently published study, renal azotemia was however diagnosed in $14 \%$ of dogs with babesiosis caused by $B$. rossi, documenting that renal azotemia is a relatively frequent complication (Defauw et al., 2018). Grading of AKI was introduced by the International Renal Interest Society (IRIS) in order to emphasize that AKI can vary from mild to severe kidney injury with overt ARF (Cowgill, 2016). Diagnosis of such milder forms of AKI is difficult, especially in the setting of canine babesiosis. At least $75 \%$ of the nephrons must be nonfunctional before the routine functional renal biomarker serum creatinine $(\mathrm{sCr})$ will increase above the upper limit of the reference interval (Braun et al., 2003; Hokamp and Nabity, 2016). In canine babesiosis, traditional assessment of renal function based on sCr is hampered in several additional ways. The presence of hemolysis, leading to elevated serum bilirubin and free hemoglobin, can result in interference with the most commonly used laboratory techniques measuring sCr (Braun et al., 2003; de Scally et al., 2004). Moreover, after experimentally infecting dogs with Babesia canis, decreasing plasma creatinine concentrations were seen, likely due to an increased plasma volume after infection (Schetters et al., 2009). Alternatively, a reduced creatinine production in sepsis and in critical illness could also explain this trend (Doi et al., 2009; Wilson et al., 2012). hampered. More sensitive alternative biomarkers are needed to evaluate AKI in canine babesiosis. Novel urinary biomarkers can detect kidney injury at an early stage and can quantify and localize injury to a specific origin (i.e., glomerular versus tubular region) (De Loor et al., 2013; Hokamp and Nabity, 2016). Early recognition of AKI using these sensitive urinary biomarkers allows earlier therapeutic 
104

105

106

107

108

109

110

111

112

113

114

115

116

117

118

119

120

121

122

123

124

125

126

127

128

129

130

131

132

133

134

135

136

137

138

that correlates strongly with glomerular filtration rate (GFR) in dogs with chronic kidney disease (CKD) (Nabity et al., 2015). In a recent study, plasma SDMA was also able to identify dogs with azotemic AKI (Dahlem et al., 2017). In humans, follow-up of renal function after an episode of AKI is advised at least in high-risk patients, because patients surviving an AKI episode have an independently increased risk to develop CKD (Coca et al., 2012; Rimes-Stigare et al., 2015; Heung et al., 2016; Vanmassenhove et al., 2018). Kidney injury biomarkers have the potential to predict renal recovery from AKI in humans (Koraishy and Coca, 2014).

The main objective of this study was to characterize AKI in dogs with babesiosis caused by B. rossi at presentation, during hospitalization, and at follow-up 1 month after treatment, comparing routine and novel biomarkers of kidney injury and dysfunction. As a secondary aim, the same biomarkers were compared between dogs with and without complicated babesiosis to determine the effect of complicated disease on kidney injury and dysfunction.

\section{Materials and methods}

\subsection{Study population and design}

Client-owned dogs presenting with babesiosis to the Onderstepoort Veterinary Academic Hospital (OVAH, University of Pretoria, South Africa) were prospectively evaluated for inclusion in this observational study between December 2015 and January 2017. Initial diagnosis of babesiosis was made based on presence of compatible clinical signs and detection of large Babesia species on a stained blood smear. The causative agent was later confirmed as B. rossi based on polymerase chain reaction (PCR) and reverse line blot (RLB) (Matjila et al., 2008). During the same period, clinically healthy client-owned control dogs of comparable age and weight presenting to the OVAH for vaccination, routine sterilization, or blood donation, were also recruited. The study was approved by the Animal Ethics Committee of the University of Pretoria (protocol numbers V034-14 and V098-15) and informed owner consent was obtained.

In the dogs with babesiosis, a thorough medical history, physical examination, complete blood count, biochemistry profile (sCr, serum urea, glucose, total protein, albumin, globulin, alanine aminotransferase, alkaline phosphatase, total bilirubin, and phosphate) and a complete routine urinalysis (including urinary protein to creatinine ratio (UPC) and aerobic bacterial culture) was performed at presentation ( $\mathrm{T}_{0}$ ), also to exclude concurrent diseases. Blood pressure was measured in all dogs with babesiosis using oscillometry. Blood and urine samples were collected before any therapy, especially fluid therapy, was initiated. Control dogs were considered healthy based on the absence of clinically relevant abnormalities in the medical history, physical examination, complete blood count, biochemistry profile, and a complete routine urinalysis (including a UPC $<0.5$ ). Urine was collected by cystocentesis in all dogs.

In both groups, dogs with a urinary tract infection (UTI) at $\mathrm{T}_{0}$ were excluded based on bacterial growth detection in urine within 3 days of incubation. Dogs with suspected pre-existing CKD were excluded as best as possible based on a thorough history and physical examination.

Dogs were also excluded if wounds or trauma were evident, if concurrent neoplastic, inflammatory, cardiac disease, or other infections were diagnosed, or if they were known to have been treated with anti-inflammatory medication within 4 weeks prior to presentation. PCR/RLB batch analysis for babesiosis and ehrlichiosis was performed in both groups. Dogs with babesiosis were included only after confirmation of B. rossi infection and after exclusion of Babesia vogeli and/or concurrent Ehrlichia canis infection. Control dogs that tested positive for any Babesia species or E. canis were excluded.

In dogs with babesiosis, follow-up blood and urine samples were obtained 24 hours after presentation ( $\left.\mathrm{T}_{24 \mathrm{~h}}\right)$ and 1 month after treatment $\left(\mathrm{T}_{1 \mathrm{~m}}\right)$ whenever possible. Follow-up samples at $\mathrm{T}_{24 \mathrm{~h}}$ were only collected in dogs that were hospitalized because of disease severity. At $\mathrm{T}_{24 \mathrm{~h}}$ 
sCr was measured and a complete urinalysis was performed. A complete blood count, biochemistry profile and complete urinalysis as well as PCR/RLB for babesiosis and ehrlichiosis was repeated at $\mathrm{T}_{1 \mathrm{~m}}$.

141 All infected dogs received antibabesial treatment with diminazene aceturate (Berenil RTU, Intervet, Kempton Park, South Africa) at 3.5

$142 \mathrm{mg} / \mathrm{kg}$, and standard supportive care such as packed red blood cells and intravenous fluid therapy as needed. Any complications were

143 treated as needed by the attending clinician. Dogs were diagnosed with complicated babesiosis when at least one of the following

144 complications were diagnosed either at presentation or during hospitalization. These criteria were based on previous publications defining

145 complicated babesiosis and severe falciparum malaria (Jacobson and Clark, 1994; Jacobson, 2006; WHO, 2014). Dogs were considered to

146 be collapsed at presentation when they were unable to walk unaided. Hypotension was defined as a mean arterial pressure $<80 \mathrm{mmHg}$.

147 Severe anemia was diagnosed when the hematocrit was $<0.15$. Hemoconcentration was defined as a hematocrit $>0.37$ in combination with

148 signs of intravascular hemolysis (macroscopic hemoglobinuria and/or hemoglobinemia). Secondary IMHA was diagnosed based on a 149 positive in-saline agglutination test. Diagnosis and grading of AKI was based on IRIS criteria (Cowgill, 2016). Grade I AKI was defined as a progressive increase in $\mathrm{sCr}$ (of at least $26.4 \mu \mathrm{mol} / \mathrm{L}$ ) within the non-azotemic range and within $48 \mathrm{~h}$. Hypoglycemia was diagnosed when blood glucose was $<3.3 \mathrm{mmol} / \mathrm{L}$. Jaundice was diagnosed when severe hyperbilirubinemia was present (total bilirubin $>50 \mu \mathrm{mol} / \mathrm{L}$ ). Cerebral babesiosis was defined as presence of neurological signs not attributable to another cause, such as hypoglycemia. Clinical diagnosis of ARDS was based on presence of dyspnea and variably on arterial blood gas analysis, radiological evidence of pulmonary edema, and exclusion of other causes of pulmonary edema. Outcome categories were short-term survival (i.e., survival until discharge) or death/euthanasia due to poor prognosis (as deemed by the attending clinician), and long-term survival (i.e., survival based on telephone contact with owners 4-5 weeks after presentation).

\subsection{Sample handling and laboratory analyses}

159 Automated analyzers were used for complete blood count (ADVIA 2120, Siemens, Munich, Germany) and serum biochemistry analysis (Cobas Integra 400 plus, Roche Diagnostics, Mannheim, Germany). Serum creatinine was determined using a modified Jaffe method. A complete urinalysis, including dipstick (Combur 9 Test ${ }^{\circledR}$, Roche Diagnostics, Germany), urine specific gravity (USG), microscopic sediment analysis within 60 minutes, UPC, and aerobic bacterial culture, was performed. After urine collection, $0.5 \mathrm{ml}$ of uncentrifuged urine was submitted for aerobic bacterial culture. After centrifugation of remaining urine ( 3 minutes at $447 \mathrm{xg}$ ), the supernatant was used for dipstick analysis, measurement of USG by refractometry (Atago MASTER-SUR/NM, Atago Co., Tokyo, Japan), and measurement of UPC. Remaining supernatant was divided into aliquots of $0.5-1 \mathrm{ml}$, followed by storage at $-80^{\circ} \mathrm{C}$. Remaining plasma and serum of all samples were also stored at $-80^{\circ} \mathrm{C}$. PCR/RLB batch analysis was performed on stored packed blood cell pellets. Urine osmolality (uOsmol) was measured as a batch by freezing point depression within $10 \mathrm{~m}$ after collection (Micro-Osmometer Autocal Type 13/13DR, Roebling, Berlin, Germany). Osmolality of a calibration standard solution was measured in triplicate every day before sample analysis. All samples were 
173 Kidney injury biomarkers, measured by commercial immunoassays at $\mathrm{T}_{0}, \mathrm{~T}_{24 \mathrm{~h}}$, and $\mathrm{T}_{1 \mathrm{~m}}$, included urinary immunoglobulin $\mathrm{G}$ (uIgG), urinary

174 C-reactive protein (uCRP), urinary retinol-binding protein (uRBP), urinary and plasma neutrophil gelatinase-associated lipocalin (uNGAL

175 and pNGAL). Measurement of UIgG and uCRP was performed with canine sandwich enzyme-linked immunosorbent assay (ELISA) kits

176 (Immunology Consultants Laboratory, Newberg, USA), while uRBP was measured with a human sandwich ELISA kit (Immunology

177 Consultants Laboratory, Newberg, USA). Validation of the IgG, CRP, and RBP assays for use in canine urine was previously performed in

178 our laboratory (Maddens et al., 2010). Measurement of uNGAL and pNGAL was performed with a validated canine sandwich ELISA kit

179 (BioPorto Diagnostics A/S, Hellerup, Denmark). Quantification of uIgG, uCRP, uRBP, uNGAL and pNGAL by immunoassays was performed

180 as described (Defauw et al., 2012). Finally, urinary biomarker concentrations were normalized to urinary creatinine concentrations (/uCr).

181 By dividing absolute uNGAL concentrations by pNGAL concentrations, the u/pNGAL concentration ratio was calculated (Uttenthal et al.,

182 2012). The purpose of uNGAL to pNGAL normalization was to aid in the differentiation between kidney injury and non-renal increases of

183 NGAL. All analyses of kidney injury biomarkers were performed within 6 months of sample collection, except 1 sample for uIgG and 4

184 samples for uRBP that required re-analysis with another dilution. Serum SDMA (IDEXX SDMA ${ }^{\mathrm{TM}}$ Test) was measured as a novel functional renal biomarker at $\mathrm{T}_{0}, \mathrm{~T}_{24 \mathrm{~h}}$, and $\mathrm{T}_{1 \mathrm{~m}}$. Serum samples were transported on dry ice to a reference diagnostic laboratory (IDEXX Laboratories, Inc., Ludwigsburg, Germany) for batch analysis. All sSDMA samples were analyzed within 24 months after collection.

\subsection{Renal samples, light microscopy, and transmission electron microscopy}

Post-mortem kidney biopsies were obtained whenever possible. Tissue samples were fixed in 10\% buffered formalin for light microscopy (LM), and in 3\% buffered glutaraldehyde for transmission electron microscopy (TEM). Samples were processed into paraffin wax blocks for LM and resin-embedded blocks for TEM. For LM, sections were stained with hematoxylin and eosin, periodic acid-Schiff (PAS), Masson's trichrome, and periodic acid methenamine silver, according to standard procedures (Aresu et al., 2017). All sections for LM and TEM were examined by a board-certified veterinary pathologist (LA).

\subsection{Statistical analysis}

196 As the normal distribution assumption did not hold for some of the biomarkers, Mann-Whitney U tests were used to compare renal 197 biomarkers between dogs with and without babesiosis, and between dogs with complicated and uncomplicated babesiosis. Renal 198 biomarkers in B. rossi-infected dogs were compared between $\mathrm{T}_{0}, \mathrm{~T}_{24 \mathrm{~h}}$, and $\mathrm{T}_{1 \mathrm{~m}}$ using Wilcoxon signed-rank tests for paired samples. $\mathrm{P}$ values $<0.05$ were considered significant. Data were visualized through box plots. All analyses were performed using R (R version 3.3.2 Copyright (C) 2016, The R Foundation for Statistical Computing).

\section{Results}

203 3.1. Study population and clinicopathological findings

204 Forty-three dogs with babesiosis were evaluated for inclusion. Eight dogs were excluded for the following reasons: based on PCR/RLB analysis, 4 dogs tested positive for E. canis, one dog was infected with B. vogeli, and one dog was repetitively negative by PCR/RLB for 
207

208

209

210

211

212

213

214

215

216

217

218

219

220

221

222

223

224

225

226

227

228

229

230

231

232

233

234

235

236

237

238

239

240

These 3 dogs were included, because a UTI was considered unlikely, based on the absence of compatible clinical signs and of pyuria and bacteriuria on sediment analysis.

Finally, 35 of the 43 dogs with $B$. rossi infection were included. Ten dogs served as healthy controls. There was no significant age difference between dogs with babesiosis (median, 2.5 years; range, 2 months to 12.5 years) and controls ( 2 years; 4 months to 9.5 years) ( $\mathrm{P}=0.47)$. Body weight also did not differ significantly between Babesia-infected dogs (22 kg; 2.1-48 kg) and controls (28.45 kg; $4.9-61 \mathrm{~kg}$ ) (P=0.20). In the babesiosis group, there were 9 mixed breed dogs, 2 American pit bull terrier, 2 Boerboel, 2 Jack Russell terrier, 2 Labrador retriever, 2 Pekingese, 2 Weimaraner, and a single dog from other breeds. The controls consisted of 3 German shepherd, 2 mixed breed dogs, and a single dog from other breeds. Hematology and biochemistry results of both groups are summarized in Table 1 .

In the dogs with babesiosis, urine ranged from clear to very turbid and urine color ranged from yellow to dark brown. In the urinary dipstick analysis of Babesia-infected dogs, $\mathrm{pH}$ ranged from 5 to 8 , proteinuria from $1+$ to $3+$, bilirubinuria from negative to $3+$, hemoglobinuria from negative to 4+ (4+ in 30/35 dogs), while glucose and ketones were always negative. Bilirubin crystalluria was present in 9 dogs with babesiosis. Rare to large amounts of granular casts were seen in 23 Babesia-infected dogs, while rare hyaline casts were seen in 5 Babesia-infected dogs. Microscopic hematuria (i.e., >5 red blood cells/high power field) was seen in 6 Babesia-infected dogs, while pyuria (i.e., >5 white blood cells/high power field) was absent in all dogs.

\subsection{AKI biomarkers at $T_{0}$}

In Table 2, results of routine renal biomarkers ( $\mathrm{SCr}$, serum urea, USG, uOsmol, and UPC) at $\mathrm{T}_{0}$ are compared between healthy controls and dogs with babesiosis. In Fig. 1 and Table 3, results of kidney injury biomarkers (uIgG/uCr, uCRP/uCr, uRBP/uCr, uNGAL/uCr, pNGAL, $\mathrm{u} / \mathrm{pNGAL}$ ) and sSDMA at $\mathrm{T}_{0}$ are presented and compared between both groups. All kidney injury biomarkers, including UPC, were significantly higher in Babesia-infected dogs compared to healthy controls, while sCr, serum urea, USG, uOsmol, and sSDMA did not significantly differ between both groups at $\mathrm{T}_{0}$. However, when healthy controls were compared with the subgroup of dogs with uncomplicated babesiosis, sCr was significantly lower in the dogs with uncomplicated disease $(\mathrm{P}=0.009)$.

Based on the reference interval (0-14 $\mu \mathrm{g} / \mathrm{dL}$ for adult dogs, 0-16 $\mu \mathrm{g} / \mathrm{dL}$ for dogs up to $12 \mathrm{~m}), 3 / 10$ healthy dogs (30\%) and $16 / 35$ dogs with babesiosis (46\%) showed increased sSDMA concentrations. On physical examination, 7/35 dogs with babesiosis had clinical signs of dehydration, of which 4 had increased sSDMA concentrations.

\subsection{Follow-up at $T_{24 h}$ and $T_{1 m}$}

Twenty-four of the 35 dogs with babesiosis were hospitalized. At $\mathrm{T}_{24 \mathrm{~h}}$, follow-up blood and urine samples were collected in 11 dogs with babesiosis, and follow-up samples were available from 9 Babesia-treated dogs at $\mathrm{T}_{1 \mathrm{~m}}$. Seven of these dogs had follow-up samples collected at both $\mathrm{T}_{24 \mathrm{~h}}$ and $\mathrm{T}_{1 \mathrm{~m}}$. Because of a UTI, one dog at $\mathrm{T}_{24 \mathrm{~h}}$ and 2 dogs at $\mathrm{T}_{1 \mathrm{~m}}$ were excluded from urinary kidney injury biomarker analyses. One of the 9 dogs had a faintly positive PCR/RLB test for a Babesia species at $\mathrm{T}_{1 \mathrm{~m}}$. With exception of sSDMA, which was borderline increased (15 $\mu \mathrm{g} / \mathrm{dL})$, all renal biomarkers in this dog were normalized at $\mathrm{T}_{1 \mathrm{~m}}$. Short-term survival was $89 \%$ (31/35 dogs). Two dogs died spontaneously within 8 hours after admission, while 2 dogs were euthanized during hospitalization due to poor prognosis. As for longterm survival, all dogs discharged from the hospital survived, but 2 dogs were lost to follow-up. 
241 In Table 2, routine renal biomarkers results are compared between $\mathrm{T}_{0}, \mathrm{~T}_{24 \mathrm{~h}}$, and $\mathrm{T}_{1 \mathrm{~m}}$. In Fig. 1 and Table 3 , results of kidney injury

242 biomarkers and sSDMA are compared between $\mathrm{T}_{0}, \mathrm{~T}_{24 \mathrm{~h}}$, and $\mathrm{T}_{1 \mathrm{~m}}$. Results of biomarker comparison between healthy controls and Babesia-

243 treated dogs at $\mathrm{T}_{1 \mathrm{~m}}$ are also shown in Fig. 1, Tables 2 and 3.

244 At $\mathrm{T}_{24 \mathrm{~h}}, \mathrm{UPC}, \mathrm{uRBP} / \mathrm{uCr}, \mathrm{uNGAL} / \mathrm{uCr}$, and $\mathrm{u} / \mathrm{pNGAL}$ decreased significantly (Tables 2 and 3, Fig. 1). At $\mathrm{T}_{1 \mathrm{~m}}$, decreases were significant for 245 USG and all urinary kidney injury biomarkers (Tables 2 and 3, Fig.1). Significant changes in sCr, sSDMA, pNGAL, and uOsmol were not seen during follow-up at $\mathrm{T}_{24 \mathrm{~h}}$ and $\mathrm{T}_{1 \mathrm{~m}}$. At $\mathrm{T}_{1 \mathrm{~m}}$, all renal biomarkers had values not significantly different from the healthy controls, except for pNGAL which was still significantly higher in the Babesia-treated dogs. At $\mathrm{T}_{24 \mathrm{~h}}, 6 / 11$ dogs with babesiosis (55\%) had increased sSDMA concentrations, while at $\mathrm{T}_{1 \mathrm{~m}} 2 / 9 \mathrm{dogs}$ with babesiosis (22\%) had a borderline increased sSDMA (15 $\left.\mu \mathrm{g} / \mathrm{dL}\right)$.

Ten dogs were diagnosed with uncomplicated babesiosis, while at least one complication occurred in each of the remaining 25 dogs. At $\mathrm{T}_{0}$, 7 dogs were collapsed, 4 dogs were hypotensive, 9 dogs were severely anemic, 3 dogs were diagnosed with hemoconcentration, while 4 dogs presented with secondary IMHA. Based on sCr, 3 dogs were mildly azotemic and diagnosed with AKI grade II. Two of these dogs had prerenal azotemia based on presence of concentrated urine (USG $\geq 1.030 ; \mathrm{uOsmol} \geq 1110 \mathrm{mOsmol} / \mathrm{kg}$ ). The other dog was diagnosed with renal azotemia (USG 1.018; uOsmol $430 \mathrm{mOsmol} / \mathrm{kg}$ ). Four dogs were hypoglycemic, while jaundice was diagnosed in 5 dogs. Cerebral babesiosis was diagnosed in 2 dogs. At $\mathrm{T}_{24 \mathrm{~h}}, \mathrm{sCr}$ was available in 13 dogs and 5 additional cases of AKI were diagnosed ( 3 and 2 dogs with AKI grade I and II, respectively). Finally, one dog was diagnosed with ARDS during hospitalization. Of the 9 dogs with follow-up available at $\mathrm{T}_{1 \mathrm{~m}}, 2$ dogs were presented with AKI grade II at $\mathrm{T}_{0}$, while 2 dogs developed AKI grade I and one dog AKI grade II during hospitalization. In these 5 dogs diagnosed with AKI at $\mathrm{T}_{0}$ or $\mathrm{T}_{24 \mathrm{~h}}$, no evidence of kidney injury or dysfunction was detected at $\mathrm{T}_{1 \mathrm{~m}}$, except for one dog with a borderline increased sSDMA (15 $\mu \mathrm{g} / \mathrm{dL})$.

\subsection{AKI biomarkers in uncomplicated and complicated babesiosis at $T_{0}$}

264 In Table 4, results of routine renal biomarkers, kidney injury biomarkers, and sSDMA at $\mathrm{T}_{0}$ are compared between the subgroups of dogs with uncomplicated and complicated disease. Dogs with complicated babesiosis had significantly higher uIgG/uCr, uCRP/uCr, UPC, serum urea, and sSDMA, while uRBP/uCr, uNGAL/uCr, pNGAL, u/pNGAL, USG, uOsmol, and sCr did not differ significantly between groups. Increased sSDMA concentrations were seen in 14/25 dogs with complicated disease (56\%), while 2/10 dogs with uncomplicated

271 Within 6 hours after spontaneous death, kidney samples were obtained from 2 dogs with complicated babesiosis. Dog 1 was a 2-month-

272 old male intact Irish wolfhound, presenting with peracute cerebral babesiosis. The first clinical signs (lethargy and vomiting) were noticed

273 a couple of hours before presentation. Serum creatinine was $34 \mu \mathrm{mol} / \mathrm{L}$, sSDMA was $12 \mu \mathrm{g} / \mathrm{dL}$, and UPC was 2.76 . Urine sediment showed

274 mild microscopic hematuria and absence of casts. Kidney injury biomarkers were as followed: uIgG/uCr: $126.03 \mathrm{mg} / \mathrm{g}$, uCRP/uCr: 0.21

$275 \mathrm{mg} / \mathrm{g}$, uRBP/uCr: $0.26 \mathrm{mg} / \mathrm{g}$, uNGAL/uCr: $77.44 \mu \mathrm{g} / \mathrm{g}$, pNGAL: $8.57 \mathrm{ng} / \mathrm{mL}$, u/pNGAL: 19.98. A moderate increase of mesangial matrix was 
276

277

278

279

280

281

282

283

284

285

286

287

288

289

290

291

292

293

294

295

296

297

298

299

300

301

302

303

304

305

306

307

308

309

310

seen at glomerular level, but the most significant lesions were evident at tubular level. Multifocally, tubules were necrotic and intratubular protein casts (PAS-positive) were identified. Many tubules showed degenerating epithelial cells, characterized by cellular swelling and cytoplasmic rarefaction. Focal tubular epithelial cells showed loss of brush border. The histological diagnosis was compatible with a multifocal, moderate acute tubular necrosis (ATN) and degeneration further confirmed by TEM.

Dog 2 was a 10-year-old male intact mixed breed dog, presenting with a 4-day history of anorexia and lethargy. The dog was collapsed, hypotensive, and severely hypoglycemic at presentation. Serum creatinine was $101 \mu \mathrm{mol} / \mathrm{L}$, sSDMA was $20 \mu \mathrm{g} / \mathrm{dL}$, and UPC was 7.73. Urine was dark brown and turbid, sediment showed a large amount of granular casts. Kidney injury biomarkers were as followed: $\mathrm{uIgG/uCr:}$ 1708.90 mg/g, uCRP/uCr: 10.55 mg/g, uRBP/uCr: 7.71 mg/g, uNGAL/uCr: 2354.03 g/g, pNGAL: 121.77 ng/mL, u/pNGAL: 34.02. A moderate increase of mesangial matrix was observed, but no other glomerular or tubular alterations were evident both at LM and TEM.

\section{Discussion}

In this population of dogs with babesiosis caused by B. rossi, our selected kidney injury biomarkers were all able to detect kidney injury at an earlier stage compared to both serum functional renal biomarkers (i.e., sCr and sSDMA). Earlier recognition of AKI could ultimately lead to an improved outcome by allowing prompt therapeutic interventions and appropriate monitoring. Moreover, there was no evidence of active ongoing kidney injury at $\mathrm{T}_{1 \mathrm{~m}}$, suggesting a transient nature of kidney injury in most dogs after successful treatment. All infected dogs, irrespective of disease severity (uncomplicated versus complicated babesiosis), suffered from comparable kidney injury based on tubular injury biomarker concentrations. However, sSDMA was significantly higher in dogs with complicated babesiosis, suggesting that renal function should be monitored more closely in this subgroup of critically ill dogs.

All kidney injury biomarkers were significantly higher in Babesia-infected dogs compared to healthy controls, while neither of the functional renal biomarkers differed significantly. Increased urinary concentrations of glomerular and tubular injury biomarkers (i.e., uIgG, uCRP and uRBP) in dogs with uncomplicated B. rossi infection have been documented in a previous study from our group (Defauw et al., 2012). Similar findings were observed in 2 recently published papers on B. canis infections in Europe, where glomerular and tubular injury were also detected based on increased urinary kidney injury biomarkers (Winiarczyk et al., 2017; Kuleš et al., 2018). Another study involving B. rossi demonstrated, based on urine sediment analysis, UPC and urinary enzyme concentrations, that milder manifestations of kidney injury are much more common than overt ARF (Lobetti and Jacobson, 2001). Based on these studies, B. rossi-induced AKI manifests itself primarily by kidney injury rather than by loss of function. However, an important limitation is that the functional renal biomarkers used in all these studies are either insensitive ( $\mathrm{sCr}$ ) or not fully validated for use in canine AKI (sSDMA) (Hokamp and Nabity, 2016; Dahlem et al., 2017). Indeed, sSDMA correlates well to GFR in dogs with CKD (Nabity et al., 2015), but the relation between sSDMA and GFR in dogs with AKI remains unknown (Dahlem et al., 2017). A recent study demonstrated that plasma SDMA was an excellent biomarker to id entify azotemic AKI in dogs, but dogs with non-azotemic AKI grade I were not evaluated (Dahlem et al., 2017).

In the current study NGAL was used for the first time in Babesia-infected dogs as a sensitive biomarker of tubular injury (Segev et al., 2013; Nabity et al., 2015). In a previous study, uNGAL was also increased in dogs with AKI Grade I, showing its potential as a sensitive biomarker of milder forms of AKI (Segev et al., 2013). However, in human medicine (Mårtensson and Bellomo, 2014) and more recently also in veterinary medicine (Cortellini et al., 2015; Cobrin et al., 2016), the specificity of NGAL for AKI has been questioned. It is important to realize that competition for tubular reabsorption between NGAL and other filtered proteins could result in increased uNGAL and $u / p N G A L$ 
311 ratio as well, independent of the presence of tubular injury (Nejat et al., 2012). During systemic inflammation, pNGAL and uNGAL can also

312 increase due to increased synthesis from non-renal tissues and increased release from circulating neutrophils (Mårtensson and Bellomo,

313 2014). Because both systemic and urinary NGAL concentrations were measured in this study, the impact of systemically originating NGAL

314 in canine babesiosis can be assessed to a certain extent. The $\mathrm{u} / \mathrm{pNGAL}$ ratio was also calculated in the current study to aid in the

315 differentiation between kidney injury and non-renal causes of increased UNGAL, because relatively higher concentrations of uNGAL

316 compared to pNGAL are expected during kidney injury (Uttenthal et al., 2012). To the authors' knowledge, this ratio has never been used

317 in dogs before, but it has been applied in several other species, including humans (Bagshaw et al., 2010; Nielsen et al., 2010; Kaucsár et al.,

318 2016). The $\mathrm{u} / \mathrm{pNGAL}$ ratio does however not correct for variations in the urinary flow rate. When NGAL concentrations in the current study

319 were compared to NGAL concentrations reported in a study with septic dogs (Cortellini et al., 2015), the median systemic NGAL

320 concentration was 5-6 times higher in dogs with bacterial sepsis compared to dogs with babesiosis, while the median uNGAL concentration

321 was very similar in both studies. The relative impact of systemically orginating NGAL on the total uNGAL concentration therefore seems

322 less pronounced in dogs with babesiosis, which is suggestive of more kidney injury-specific increases of uNGAL in dogs with babesiosis

323 compared to septic dogs. The highly increased u/pNGAL ratio at $\mathrm{T}_{0}$ is also supportive of kidney injury-specific increases of uNGAL in $B$.

324 rossi-infected dogs. Why NGAL appears to be more specific for kidney injury in the current study compared to other studies (Cortellini et

325 al., 2015; Cobrin et al., 2016), may be explained by the fact that all dogs in our study were infected with the same organism which causes a

326 disease in which neutrophilia is uncommon (Scheepers et al., 2011), thus reducing the impact of NGAL originating from circulating

327 neutrophils (Mårtensson and Bellomo, 2014).

328 Several studies in humans demonstrated an increased risk for CKD and long-term mortality after an AKI episode (Coca et al., 2009; Heung et al., 2016; Vanmassenhove et al., 2018). This increased risk was documented even after milder forms of AKI with apparent fast recovery (Coca et al., 2009; Heung et al., 2016). Using specific biomarkers to detect active ongoing kidney injury might help to predict which dogs are at risk of developing CKD (Cowgill et al., 2016). Therefore, the concentration of renal biomarkers was followed over time in a subset of B. rossi-infected dogs to assess the reversibility of kidney injury and dysfunction.

333 At $\mathrm{T}_{24 \mathrm{~h}}$, all urinary biomarkers of tubular injury (i.e., uRBP and uNGAL) and u/pNGAL decreased significantly more rapidly compared to

334 those of glomerular injury (i.e., uIgG and uCRP), whose decreases only became significant by $\mathrm{T}_{1 \mathrm{~m}}$. This finding indicates that ongoing tubular

335 injury quickly abates, while uIgG and uCRP concentrations take longer to decrease likely due to ongoing glomerular injury and persistently increased serum concentrations of these proteins. Several studies demonstrated increased serum CRP concentrations in canine babesiosis, and in 2 of these only a mild decrease was seen after 24 hours (Matijatko et al., 2007; Köster et al., 2009). Even though glomerular barrier injury must occur before UIgG and uCRP can be detected (D'Amico and Bazzi, 2003), the extent to which variations in their serum concentrations influence these urinary concentrations is unknown. With exception of pNGAL, all kidney injury biomarkers were not significantly different at $\mathrm{T}_{1 \mathrm{~m}}$ compared to healthy controls, suggesting that there was no evidence of active ongoing kidney injury in these dogs 1 month after Babesia-induced AKI. This finding argues for a transient nature of the kidney insult caused by acute $B$. rossi infections. The reason for increased pNGAL concentrations at $\mathrm{T}_{1 \mathrm{~m}}$ is unclear. It is very unlikely that tubular injury was still ongoing, considering the low uNGAL concentrations at that time. Therefore, non-renal causes of increased pNGAL are likely, such as the presence of an unrelated inflammatory condition. Based on sCr and sSDMA, there was no evidence of decreased renal function at $\mathrm{T}_{1 \mathrm{~m}}$. However, sensitivity for 
borderline increased sSDMA at $\mathrm{T}_{1 \mathrm{~m}}(15 \mu \mathrm{g} / \mathrm{dL})$. One of these dogs had a $\mathrm{SCr}$ of $110 \mu \mathrm{mol} / \mathrm{L}$ and highly concentrated urine, but the other dog ( $\mathrm{sCr}$ of $93 \mu \mathrm{mol} / \mathrm{L}$ ) had a relatively low urinary concentration at $\mathrm{T}_{1 \mathrm{~m}}$ (USG 1.022; uOsmol $776 \mathrm{mOsmol} / \mathrm{kg}$ ). Based on the normalization of all kidney injury biomarkers, there was no evidence of active kidney injury in these 2 dogs at $\mathrm{T}_{1 \mathrm{~m}}$. Whether the dog with relatively low urinary concentration had early-stage CKD, is uncertain. Although there was no evidence of active kidney injury based on the investigated kidney injury biomarkers in this small number of dogs included at $\mathrm{T}_{1 \mathrm{~m}}$, a long-term follow-up study, including a larger numbers dogs followed over a longer time period, would be necessary to fully clarify if there is an increased risk for the development of CKD related to early-life Babesia-induced AKI. In dogs with complicated disease, both of the glomerular injury biomarkers were significantly higher, while none of the tubular injury biomarkers differed significantly between uncomplicated and complicated cases. More severe glomerular injury and/or higher systemic concentrations of IgG and CRP in complicated cases could explain these findings. Significantly higher sSDMA concentrations in complicated cases could be explained by a decreased GFR caused by renal hypoperfusion and/or intrinsic AKI (Choi et al., 2017; Dahlem et al., 2017). Renal hypoperfusion, for example due to dehydration, is unlikely to be the main reason of higher sSDMA concentrations in most complicated cases when urinary concentrating ability is taken into account, as a higher uOsmol would be expected in that situation. However, uOsmol was close to being significantly lower in dogs with complicated babesiosis. Overall, these findings confirm that all dogs with babesiosis caused by B. rossi, irrespective of disease severity, suffer kidney injury based on the urinary biomarker results, while sSDMA and uOsmol concentrations suggest that the infection also induces significant loss of renal function in many dogs with complicated babesiosis. Moderate to severe ATN and degeneration, without significant morphological lesions at glomerular level, were identified in dog 1. These findings are consistent with the sparse literature available on renal histological changes in canine babesiosis caused by large species which document mainly tubular lesions (Maegraith et al., 1957; Hildebrandt, 1981; Irwin and Hutchinson, 1991; Máthé et al., 2007). Unexpectedly, mild morphological changes were found in $\operatorname{dog} 2$, whose clinical presentation and presence of highly elevated kidney injury biomarkers were suggestive of clinically significant AKI. Animals in experimental studies and people with hypoxic and/or septic AKI also often lack histological ATN, either because of its absence or because of mild, focally distributed lesions (Langenberg et al., 2008; Heyman et al., 2010; Takasu et al., 2013). It is well documented in human nephropathology that renal tissue injury is often limited and focal when a clinical diagnosis of AKI/ATN is made (Heyman et al., 2010). Consequently, kidney injury biomarkers could lack accuracy for diagnosing histological ATN, which has already been documented in humans (Moledina et al., 2017). Considering only 2 dogs had histological evaluations performed in this study, additional research is necessary to clarify the link between kidney injury biomarkers and histological findings.

374 Considering that degeneration and necrosis of the tubular cells are amongst the most common histological changes seen in kidneys of dogs infected with large Babesia spp. (Maegraith et al., 1957; Irwin and Hutchinson, 1991; Máthé et al., 2007), higher concentrations of tubular injury biomarkers were anticipated in dogs with complicated disease. Rather unexpectedly, these biomarkers did not differ significantly between uncomplicated and complicated cases, while the functional renal biomarker sSDMA was significantly higher in complicated cases. 
possible influence of the criteria defining uncomplicated versus complicated disease, and the limited information available for sSDMA as a functional renal biomarker in canine AKI (Dahlem et al., 2017). The presence of a type II error to detect differences in tubular injury biomarker concentrations between uncomplicated and complicated cases can also not be excluded. For practical reasons, follow-up at $\mathrm{T}_{24 \mathrm{~h}}$ and $\mathrm{T}_{1 \mathrm{~m}}$ was lacking for many cases. Some complications such as AKI grade $\mathrm{I}$, as well as statistically significant differences might have been missed by this low number of dogs with follow-up. However, confirmation of full clinical recovery was obtained by telephone contact for all discharged dogs. Only dogs hospitalized because of disease severity had follow-up samples at $\mathrm{T}_{24 \mathrm{~h}}$, leading to a sampling bias. Additional AKI diagnoses were made in $5 / 13$ dogs with sCr follow-up at $\mathrm{T}_{24 \mathrm{~h}}$, which is likely to be an overestimation due to this bias.

\section{Conclusions}

Dogs with babesiosis caused by B. rossi showed transient kidney injury, which was detected by all kidney injury biomarkers, yet remained undetected by routine and novel functional biomarkers. Nonetheless, our results also suggest that dogs with complicated babesiosis are more likely to present with significant loss of renal function. Therefore, adequate supportive therapy and monitoring of renal function during and after treatment is strongly advised for all dogs with babesiosis caused by B. rossi, even when no evidence of AKI is present based on routine $\mathrm{sCr}$ measurements.

\section{Declarations of interest}

\section{Acknowledgements}

This work was supported by the National Research Foundation of South Africa that funded a part of the sample collections and laboratory analyses (grant number CPRR13080726333). The authors would like to thank the staff of the clinical pathology laboratory, Department of the Companion Animal Clinical Studies, Faculty of Veterinary Science, University of Pretoria, Marizelle de Clercq for her assistance in recruiting healthy control dogs and the shipments, and the final year veterinary students and nurses who assisted during sample collections. The authors would also like to acknowledge Kristel Demeyere for her laboratory assistance.

\section{References}

Apanaskevich, D.A., Horak, I.G., Camicas, J.L., 2007. Redescription of Haemaphysalis (Rhipistoma) elliptica (Koch, 1844), an old taxon of the 
416

417

418

419

420

421

422

423

424

425

426

427

428

429

430

431

432

433

434

435

436

437

438

439

440

441

442

443

444

445

446

447

448

449

450

Braun, J.P., Lefebvre, H.P., Watson, A.D., 2003. Creatinine in the dog: a review. Vet. Clin. Pathol. 32, 162-179.

Choi, W., Kang, J.-H., Woo, S., Son, P., Yun, J., Sung, C.-J., Kim, H., Kang, B.-T., Yang, M.-P., 2017. Serum concentrations of symmetric dimethylarginine in dogs with dehydration. J. Vet. Intern. Med. 31, 1324-1325 (abstract).

Cobrin, A.R., Blois, S.L., Abrams-Ogg, A.C., Kruth, S.A., Dewey, C., Holowaychuk, M.K., Gauthier, V., 2016. Neutrophil gelatinase-associated lipocalin in dogs with chronic kidney disease, carcinoma, lymphoma and endotoxaemia. J. Small Anim. Pract. 57, 291-298.

Coca, S.G., Yusuf, B., Shlipak, M.G., Garg, A.X., Parikh, C.R., 2009. Long-term risk of mortality and other adverse outcomes after acute kidney injury: a systematic review and meta-analysis. Am. J. Kidney Dis. 53, 961-973.

Coca, S.G., Singanamala, S., Parikh, C.R., 2012. Chronic kidney disease after acute kidney injury: a systematic review and meta-analysis. Kidney Int. 81, 442-448.

Cortellini, S., Pelligand, L., Syme, H., Chang, Y.M., Adamantos, S., 2015. Neutrophil gelatinase-associated lipocalin in dogs with sepsis undergoing emergency laparotomy: a prospective case-control study. J. Vet. Intern. Med. 29, 1595-1602.

Cowgill, L.D., 2016. IRIS Grading of Acute Kidney Injury (AKI). http://www.iris-kidney.com/guidelines/grading.html (Accessed 17 January 2019).

Cowgill, L.D., Polzin, D.J., Elliott, J., Nabity, M.B., Segev, G., Grauer, G.F., Brown, S., Langston, C., van Dongen, A.M., 2016. Is progressive chronic kidney disease a slow acute kidney injury? Vet. Clin. North Am. Small Anim. Pract. 46, 995-1013.

D'Amico, G., Bazzi, C., 2003. Pathophysiology of proteinuria. Kidney Int. 63, 809-825.

Dahlem, D.P., Neiger, R., Schweighauser, A., Francey, T., Yerramilli, M., Obare, E., Steinbach, S.M.L., 2017. Plasma symmetric dimethylarginine concentration in dogs with acute kidney injury and chronic kidney disease. J. Vet. Intern. Med. 31, 799-804.

De Loor, J., Daminet, S., Smets, P., Maddens, B., Meyer, E., 2013. Urinary biomarkers for acute kidney injury in dogs. J. Vet. Intern. Med. 27, 998-1010.

de Scally, M.P., Lobetti, R.G., Reyers, F., Humphris, D., 2004. Are urea and creatinine values reliable indicators of azotaemia in canine babesiosis? J. S. Afr. Vet. Assoc. 75, 121-124.

Defauw, P., Schoeman, J.P., Smets, P., Goddard, A., Meyer, E., Liebenberg, C., Daminet, S., 2012. Assessment of renal dysfunction using urinary markers in canine babesiosis caused by Babesia rossi. Vet. Parasitol. 190, 326-332.

Defauw, P., Daminet, S., Leisewitz, A.L., Goddard, A., Paepe, D., Duchateau, L., Schoeman, J.P., 2018. Renal azotemia and associated clinical and laboratory findings in dogs with Babesia rossi infection. Vet. Parasitol. 260, 22-29.

Doi, K., Yuen, P.S., Eisner, C., Hu, X., Leelahavanichkul, A., Schnermann, J., Star, R.A., 2009. Reduced production of creatinine limits its use as marker of kidney injury in sepsis. J. Am. Soc. Nephrol. 20, 1217-1221.

Goddard, A., Leisewitz, A.L., Kjelgaard-Hansen, M., Kristensen, A.T., Schoeman, J.P., 2016. Excessive pro-inflammatory serum cytokine concentrations in virulent canine babesiosis. PLoS One 11, e0150113.

Heung, M., Steffick, D.E., Zivin, K., Gillespie, B.W., Banerjee, T., Hsu, C.Y., Powe, N.R., Pavkov, M.E., Williams, D.E., Saran, R., Shahinian, V.B., 2016. Acute kidney injury recovery pattern and subsequent risk of CKD: an analysis of Veterans Health Administration data. Am. J. Kidney Dis. 67, 742-752.

Heyman, S.N., Rosenberger, C., Rosen, S., 2010. Experimental ischemia-reperfusion: biases and myths-the proximal vs. distal hypoxic tubular injury debate revisited. Kidney Int. 77, 9-16. 
451

452

453

454

455

456

457

458

459

460

461

462

463

464

465

466

467

468

469

470

471

472

473

474

475

476

477

478

479

480

481

482

483

484

Hildebrandt, P.K., 1981. The organ and vascular pathology of babesiosis. In: Ristic, M., Kreier, J.P. (Eds), Babesiosis. Academic Press, New York, pp. 459-473.

Hokamp, J.A., Nabity, M.B., 2016. Renal biomarkers in domestic species. Vet. Clin. Pathol. 45, 28-56.

Irwin, P.J., Hutchinson, G.W., 1991. Clinical and pathological findings of Babesia infection in dogs. Aust. Vet. J. 68, $204-209$.

Jacobson, L.S., Clark, I.A., 1994. The pathophysiology of canine babesiosis: new approaches to an old puzzle. J. S. Afr. Vet. Assoc. 65, 134145.

Jacobson, L.S., 2006. The South African form of severe and complicated canine babesiosis: clinical advances 1994-2004. Vet. Parasitol. 138, $126-139$.

Kaucsár, T., Godó, M., Révész, C., Kovács, M., Mócsai, A., Kiss, N., Albert, M., Krenács, T., Szénási, G., Hamar, P., 2016. Urine/plasma neutrophil gelatinase associated lipocalin ratio is a sensitive and specific marker of subclinical acute kidney injury in mice. PLoS One 11, e0148043.

Keller, N., Jacobson, L.S., Nel, M., de Clerq, M., Thompson, P.N., Schoeman, J.P., 2004. Prevalence and risk factors of hypoglycemia in virulent canine babesiosis. J. Vet. Intern. Med. 18, 265-270.

Koraishy, F.M., Coca, S.G., 2014. Can we predict recovery from severe acute kidney injury with biomarkers? Semin. Dial. 27, $236-239$.

Köster, L.S., Van Schoor, M., Goddard, A., Thompson, P.N., Matjila, P.T., Kjelgaard-Hansen, M., 2009. C-reactive protein in canine babesiosis caused by Babesia rossi and its association with outcome. J. S. Afr. Vet. Assoc. 80, 87-91.

Köster, L.S., Steiner, J.M., Suchodolski, J.S., Schoeman, J.P., 2015. Serum canine pancreatic-specific lipase concentrations in dogs with naturally occurring Babesia rossi infection. J. S. Afr. Vet. Assoc. 86, E1-E7.

Kuleš, J., Bilić, P., Beer Ljubić, B., Gotić, J., Crnogaj, M., Brkljačić, M., Mrljak, V., 2018. Glomerular and tubular kidney damage markers in canine babesiosis caused by Babesia canis. Ticks Tick Borne Dis. 9, 1508-1517.

Langenberg, C., Bagshaw, S.M., May, C.N., Bellomo, R., 2008. The histopathology of septic acute kidney injury: a systematic review. Crit. Care 12, R38.

Lewis, B.D., Penzhorn, B.L., Lopez-Rebollar, L.M., De Waal, D.T., 1996. Isolation of a South African vector-specific strain of Babesia canis. Vet. Parasitol. 63, 9-16.

Lobetti, R.G., Jacobson, L.S., 2001. Renal involvement in dogs with babesiosis. J. S. Afr. Vet. Assoc. 72, $23-28$.

Maddens, B.E., Daminet, S., Demeyere, K., Demon, D., Smets, P., Meyer, E., 2010. Validation of immunoassays for the candidate renal markers C-reactive protein, immunoglobulin G, thromboxane B2 and retinol binding protein in canine urine. Vet. Immunol. Immunopathol. 134, $259-264$

Maegraith, B., Gilles, H.M., Devakul, L., 1957. Pathological processes in Babesia canis infections. Z. Tropenmed. Parasitol. 8, 485-514.

Mårtensson, J., Bellomo, R., 2014. The rise and fall of NGAL in acute kidney injury. Blood Purif. 37, 304-310.

Máthé, A., Dobos-Kovács, M., Vörös, K., 2007. Histological and ultrastructural studies of renal lesions in Babesia canis infected dogs treated with imidocarb. Acta Vet. Hung. 55, 511-523.

Matijatko, V., Mrljak, V., Kis, I., Kucer, N., Forsek, J., Zivicnjak, T., Romić, Z., Simec, Z., Ceron, J.J., 2007. Evidence of an acute phase response in dogs naturally infected with Babesia canis. Vet. Parasitol. 144, 242-250. 
485

486

487

488

489

490

491

492

493

494

495

496

497

498

499

500

501

502

503

504

505

506

507

508

509

510

511

512

513

514

515

516

517

518

Matjila, P.T., Leisewitz, A.L., Jongejan, F., Penzhorn, B.L., 2008. Molecular detection of tick-borne protozoal and ehrlichial infections in domestic dogs in South Africa. Vet. Parasitol. 155, 152-157.

Mitchell, P., 2015. Did disease constrain the spread of domestic dogs (Canis familiaris) into Sub-Saharan Africa? Azania 50, 92-135.

Moledina, D.G., Hall, I.E., Thiessen-Philbrook, H., Reese, P.P., Weng, F.L., Schröppel, B., Doshi, M.D., Wilson, F.P., Coca, S.G., Parikh, C.R., 2017. Performance of serum creatinine and kidney injury biomarkers for diagnosing histologic acute tubular injury. Am. J. Kidney Dis. 70, 807-816.

Nabity, M.B., Lees, G.E., Boggess, M.M., Yerramilli, M., Obare, E., Rakitin, A., Aguiar, J., Relford, R., 2015. Symmetric dimethylarginine assay validation, stability, and evaluation as a marker for the early detection of chronic kidney disease in dogs. J. Vet. Intern. Med. 29, 10361044.

Nejat, M., Hill, J.V., Pickering, J.W., Edelstein, C.L., Devarajan, P., Endre, Z.H., 2012. Albuminuria increases cystatin C excretion: implications for urinary biomarkers. Nephrol. Dial. Transplant. 27 (Suppl 3), 96-103.

Nielsen, S.E., Schjoedt, K.J., Astrup, A.S., Tarnow, L., Lajer, M., Hansen, P.R., Parving, H.H., Rossing, P., 2010. Neutrophil gelatinase-associated lipocalin (NGAL) and kidney injury molecule 1 (KIM1) in patients with diabetic nephropathy: a cross-sectional study and the effects of lisinopril. Diabet. Med. 27, 1144-1150.

Penzhorn, B.L., 2011. Why is Southern African canine babesiosis so virulent? An evolutionary perspective. Parasit. Vectors 4, 51.

Penzhorn, B.L., Vorster, I., Harrison-White, R.F., Oosthuizen, M.C., 2017. Black-backed jackals (Canis mesomelas) are natural hosts of Babesia rossi, the virulent causative agent of canine babesiosis in sub-Saharan Africa. Parasit. Vectors 10, 124.

Rimes-Stigare, C., Frumento, P., Bottai, M., Mårtensson, J., Martling, C.R., Walther, S.M., Karlström, G., Bell, M., 2015. Evolution of chronic renal impairment and long-term mortality after de novo acute kidney injury in the critically ill; a Swedish multi-centre cohort study. Crit. Care 19, 221.

Scheepers, E., Leisewitz, A.L., Thompson, P.N., Christopher, M.M., 2011. Serial haematology results in transfused and non-transfused dogs naturally infected with Babesia rossi. J. S. Afr. Vet. Assoc. 82, 136-143.

Schetters, T.P., Kleuskens, J.A., Van De Crommert, J., De Leeuw, P.W., Finizio, A.L., Gorenflot, A., 2009. Systemic inflammatory responses in dogs experimentally infected with Babesia canis; a haematological study. Vet. Parasitol. 162, 7-15.

Segev, G., Palm, C., LeRoy, B., Cowgill, L.D., Westropp, J.L., 2013. Evaluation of neutrophil gelatinase-associated lipocalin as a marker of kidney injury in dogs. J. Vet. Intern. Med. 27, 1362-1367.

Takasu, O., Gaut, J.P., Watanabe, E., To, K., Fagley, R.E., Sato, B., Jarman, S., Efimov, I.R., Janks, D.L., Srivastava, A., Bhayani, S.B., Drewry, A., Swanson, P.E., Hotchkiss, R.S., 2013. Mechanisms of cardiac and renal dysfunction in patients dying of sepsis. Am. J. Respir. Crit. Care Med. 187, 509-517.

Uilenberg, G., Franssen, F.F., Perié, N.M., Spanjer, A.A., 1989. Three groups of Babesia canis distinguished and a proposal for nomenclature. Vet. Q. 11, 33-40.

Uttenthal, L.O., Bangert, K., inventors; BioPorto Diagnostics A/S, assignee; 2012 Nov 20. Diagnostic test for renal injury. United States patent US 8313919.

Vanmassenhove, J., Vanholder, R., Lameire, N., 2018. Points of concern in post acute kidney injury management. Nephron 138, 92-103. 
519

520

521

522

523

524

525

526

527

528

529

530

531

532

533

534

535

536

537

538

539

540

541

542

543

544

545

546

547

548

549

550

551

552

553

Welzl, C., Leisewitz, A.L., Jacobson, L.S., Vaughan-Scott, T., Myburgh, E., 2001. Systemic inflammatory response syndrome and multipleorgan damage/dysfunction in complicated canine babesiosis. J. S. Afr. Vet. Assoc. 72, 158-162.

WHO, 2014. Severe malaria. Trop. Med. Int. Health 19 (Suppl 1), 7-131.

Wilson, F.P., Sheehan, J.M., Mariani, L.H., Berns, J.S., 2012. Creatinine generation is reduced in patients requiring continuous venovenous hemodialysis and independently predicts mortality. Nephrol. Dial. Transplant. 27, 4088-4094.

Winiarczyk, D., Adaszek, Ł., Bartnicki, M., Abramowicz, B., Łyp, P., Madany, J., Winiarczyk, S., 2017. Utility of urinary markers in the assessment of renal dysfunction in canine babesiosis. Tierarztl. Prax. Ausg. K. Kleintiere Heimtiere 45, 84-88. 
554

555

556

557

558

559

560

561

562

563

564

565

566

567

568

569

570

571

572

573

574

575

576

577

578

579

580

581

582

583

74

77

579
Table 1

Clinicopathological findings in 10 healthy control dogs and 35 dogs with babesiosis at presentation. All variables are expressed as median (range).

\begin{tabular}{llll}
\hline Variable (unit) & RI & H & B \\
\hline Hematocrit (L/L) & $0.37-0.55$ & $0.47(0.41-0.57)$ & $0.23(0.06-0.63)$ \\
WBC $\left(\times 10^{9} / \mathrm{L}\right)$ & $6-15$ & $8.75(7.01-12.4)$ & $6.75(1.93-22.15)$ \\
Segmented neutrophil count (x 109 /L) & $3-11.5$ & $5.77(2.94-8.93)$ & $4.32(1.17-15.28)$ \\
Band neutrophil count (x 109 /L) & $0-0.5$ & $0(0-0.12)$ & $0.16(0-2.13)$ \\
Platelet count (x 109/L) & $200-500$ & $281(142-467)$ & $11(0-166)$ \\
Total serum protein (g/L) & $56-73$ & $55(48-68)$ & $56(42-81)$ \\
Serum albumin (g/L) & $28-41$ & $35(33-38)$ & $25(14-36)$ \\
sCr ( $\mu$ mol/L) & $59-109$ & $82(47-104)$ & $63(25-132)$ \\
Serum urea (mmol/L) & $2.3-8.9$ & $5.6(2.8-8.3)$ & $7.4(3.0-27.4)$ \\
Glucose (mmol/L) & $3.3-5.5$ & $5.6(5.1-6.8)$ & $5.1(0.3-9.8)$ \\
Alanine aminotransferase (U/L) & $9-73$ & $35(22-75)$ & $30(10-124)$ \\
Alkaline phosphatase (U/L) & $20-165$ & $58(16-229)$ & $124(23-631)$ \\
Total bilirubin ( $\mu$ mol/L) & $1-6.8$ & $\mathrm{ND}$ & $8.6(1.8-248.7)$ \\
Inorganic phosphorus (mmol/L) & $0.7-2.1$ & $\mathrm{ND}$ & $1.43(0.73-3.55)$ \\
\hline
\end{tabular}

RI: reference interval; H: healthy control dogs; B: dogs with babesiosis; WBC: white blood cell count; sCr: serum creatinine; ND: not done.

76 
Table 2

585 Results of routine renal biomarkers in healthy control dogs ( $\mathrm{n}=10)$ and in dogs with babesiosis at presentation ( $\mathrm{n}=35), 24$ hours after

586 presentation ( $\mathrm{n}=11)$, and 1 month after treatment $(\mathrm{n}=9)$. All variables are expressed as median (range).

\begin{tabular}{|c|c|c|c|c|c|c|c|c|}
\hline Variable (unit) & $\mathrm{H}$ & $\mathrm{B} \mathrm{T}_{0}$ & $\mathrm{~B} \mathrm{~T}_{24 \mathrm{~h}}$ & $\mathrm{~B} \mathrm{~T}_{1 \mathrm{~m}}$ & $P a$ & $P^{\mathrm{b}}$ & $P^{c}$ & $P^{\mathrm{d}}$ \\
\hline$\overline{\mathrm{sCr}}(\mu \mathrm{mol} / \mathrm{L})$ & 82 & 63 & 67 & 76 & 0.093 & 0.18 & 0.57 & $\overline{0.54}$ \\
\hline & $(47-104)$ & $(25-132)$ & $(25-129)$ & $(46-110)$ & & & & \\
\hline Serum urea & 5.6 & 7.4 & & & 0.096 & & & \\
\hline$(\mathrm{mmol} / \mathrm{L})$ & $(2.8-8.3)$ & $(3.0-27.4)$ & & & & & & \\
\hline USG & 1.039 & 1.042 & 1.032 & 1.023 & 0.13 & 0.057 & 0.008 & 0.39 \\
\hline & $(1.017-1.050)$ & $(1.015->1.060)$ & $(1.022-1.045)$ & (1.018-1.051) & & & & \\
\hline uOsmol & 1254 & 1315 & 1062 & 927 & 0.45 & 0.15 & 0.098 & 0.35 \\
\hline (mOsmol/kg) & (579-2099) & $(430-2470)$ & (683-1704) & (676-2211) & & & & \\
\hline UPC & 0.14 & 2.63 & 1.02 & 0.11 & $<0.001$ & 0.002 & 0.016 & 0.56 \\
\hline & $(0.08-0.36)$ & $(0.34-15.42)$ & $(0.22-4.02)$ & $(0.07-0.29)$ & & & & \\
\hline
\end{tabular}

598 H: healthy control dogs; $\mathrm{B}_{\mathrm{T}}$ : dogs with babesiosis at presentation; $\mathrm{B}_{24 \mathrm{~h}}$ : dogs with babesiosis 24 hours after presentation; $\mathrm{B} \mathrm{T}_{1 \mathrm{~m}}$ : dogs

599 with babesiosis 1 month after treatment; sCr: serum creatinine; USG: urine specific gravity; uOsmol: urine osmolality; UPC: urinary

600 protein to creatinine ratio.

601 a $P$ values for the comparison between healthy control dogs and dogs with babesiosis at $\mathrm{T}_{0}$

$602{ }^{b} P$ values for the comparison between dogs with babesiosis at $\mathrm{T}_{0}$ and $\mathrm{T}_{24 \mathrm{~h}}$

603 c $P$ values for the comparison between dogs with babesiosis at $\mathrm{T}_{0}$ and $\mathrm{T}_{1 \mathrm{~m}}$

$604 \mathrm{~d} P$ values for the comparison between healthy control dogs and dogs with babesiosis at $\mathrm{T}_{1 \mathrm{~m}}$

605

606

607

608

609

610

611

612

613

614 
615

616

617

618

619

620

621

622

623

624

625

626

627

628

629

630

631

632

633

634

635

636

637

638

639

640

641

642

643

644

645

646

647

4

Table 3

Kidney injury biomarkers and serum SDMA in healthy control dogs $(n=10)$ and dogs with babesiosis at presentation (n=35), 24 hours after presentation ( $\mathrm{n}=11)$, and 1 month after treatment $(\mathrm{n}=9)$. All variables are expressed as median (range).

\begin{tabular}{|c|c|c|c|c|c|c|c|c|}
\hline Variable (unit) & $\mathrm{H}$ & $\mathrm{B} \mathrm{T}_{0}$ & $\mathrm{~B} \mathrm{~T}_{24 \mathrm{~h}}$ & $\mathrm{~B} \mathrm{~T}_{1 \mathrm{~m}}$ & $\mathrm{~Pa}$ & $\mathrm{~Pb}^{\mathrm{b}}$ & $\mathrm{P}^{\mathrm{c}}$ & $\mathrm{P}^{\mathrm{d}}$ \\
\hline $\mathrm{uIgG} / \mathrm{uCr}(\mathrm{mg} / \mathrm{g})$ & $\begin{array}{l}1.93 \\
(0.55-11.32)\end{array}$ & $\begin{array}{l}435.43 \\
(6.95-11544.30)\end{array}$ & $\begin{array}{l}190.68 \\
(4.62-1362.46)\end{array}$ & $\begin{array}{l}1.29 \\
(0.78-10.21)\end{array}$ & $<0.001$ & 0.084 & 0.016 & 0.88 \\
\hline $\mathrm{uCRP} / \mathrm{uCr}(\mathrm{mg} / \mathrm{g})$ & BDL $(n=10)$ & $\begin{array}{l}0.13(B D L-17.63) \\
\operatorname{BDL}(n=2) ; B Q L(n=\end{array}$ & $\begin{array}{l}0.14 \\
(B D L(n=1)-0.43)\end{array}$ & BDL (n=10) & $<0.001$ & 0.084 & 0.016 & 1.0 \\
\hline uRBP/uCr (mg/g) & $\begin{array}{l}0.012(B D L-0.034) \\
\operatorname{BDL}(n=2) ; B Q L(n=2)\end{array}$ & $0.92(0.26-7.71)$ & $0.74(0.020-1.16)$ & $\begin{array}{l}\text { BQL } \\
(B Q L(n=4)-0.32)\end{array}$ & $<0.001$ & 0.006 & 0.016 & 0.73 \\
\hline uNGAL/uCr $(\mu \mathrm{g} / \mathrm{g})$ & $\begin{array}{l}0.76 \\
(0.21-7.08)\end{array}$ & $\begin{array}{l}377.57 \\
(43.01-2645.43)\end{array}$ & $\begin{array}{l}246.82 \\
(29.60-782.87)\end{array}$ & $\begin{array}{l}0.30 \\
(0.08-28.06)\end{array}$ & $<0.001$ & 0.002 & 0.016 & 0.53 \\
\hline pNGAL (ng/mL) & $\begin{array}{l}6.09 \\
(2.08-12.32)\end{array}$ & $\begin{array}{l}10.71 \\
(5.95-121.77)\end{array}$ & $\begin{array}{l}15.38 \\
(6.17-29.68)\end{array}$ & $\begin{array}{l}12.09 \\
(3.52-15.41)\end{array}$ & $<0.001$ & 0.12 & 0.30 & 0.028 \\
\hline $\mathrm{u} / \mathrm{pNGAL}$ & $\begin{array}{l}0.21 \\
(0.024-3.40)\end{array}$ & $\begin{array}{l}54.77 \\
(19.98-208.54)\end{array}$ & $\begin{array}{l}31.82 \\
(1.98-68.13)\end{array}$ & $\begin{array}{l}0.28 \\
(0.032-1.51)\end{array}$ & $<0.001$ & 0.008 & 0.063 & 0.76 \\
\hline $\mathrm{sSDMA}(\mu \mathrm{g} / \mathrm{dL})$ & $\begin{array}{l}14 \\
(10-24)\end{array}$ & $\begin{array}{l}14 \\
(8-45)\end{array}$ & $\begin{array}{l}17 \\
(11-20)\end{array}$ & $\begin{array}{l}12 \\
(9-15)\end{array}$ & 0.68 & 0.88 & 0.078 & 0.12 \\
\hline
\end{tabular}

H, healthy control dogs; $B T_{0}$, dogs with babesiosis at presentation; $B T_{24 h}$, dogs with babesiosis 24 hours after presentation; B $\mathrm{T}_{1 \mathrm{~m}}$, dogs with babesiosis 1 month after treatment; uIgG, urinary immunoglobulin G; uCr, urinary creatinine; uCRP, urinary C-reactive protein; BDL, below detection limit; BQL, below quantification limit; uRBP, urinary retinol-binding protein; uNGAL, urinary neutrophil gelatinaseassociated lipocalin; pNGAL, plasma neutrophil gelatinase-associated lipocalin; u/pNGAL, urinary to plasma neutrophil gelatinaseassociated lipocalin ratio; sSDMA, serum symmetric dimethylarginine.

aP values for the comparison between healthy control dogs and dogs with babesiosis at $\mathrm{T}_{0}$ bP values for the comparison between dogs with babesiosis at $\mathrm{T}_{0}$ and $\mathrm{T}_{24 \mathrm{~h}}$ ${ }^{\mathrm{c}} \mathrm{P}$ values for the comparison between dogs with babesiosis at $\mathrm{T}_{0}$ and $\mathrm{T}_{1 \mathrm{~m}}$ ${ }^{\mathrm{d} P}$ values for the comparison between healthy control dogs and dogs with babesiosis at $\mathrm{T}_{1 \mathrm{~m}}$ 
Table 4

649 Results of routine renal biomarkers, kidney injury biomarkers, and serum SDMA in 10 dogs with uncomplicated and 25 dogs with

650 complicated babesiosis at $\mathrm{T}_{0}$. All variables are expressed as median (range).

\begin{tabular}{|c|c|c|c|}
\hline Variable (unit) & Uncomplicated disease & Complicated disease & $P$ value \\
\hline $\mathrm{sCr}(\mu \mathrm{mol} / \mathrm{L})$ & $53(32-83)$ & $70(25-132)$ & 0.27 \\
\hline Serum urea (mmol/L) & $4.8(3.0-8.2)$ & $8.6(3.4-27.4)$ & 0.007 \\
\hline USG & $1.045(1.035->1.060)$ & $1.040(1.015->1.060)$ & 0.30 \\
\hline uOsmol (mOsmol/kg) & 1509 (1199-2470) & $1236(430-2118)$ & 0.054 \\
\hline UPC & $1.24(0.34-3.79)$ & $3.18(0.51-15.42)$ & 0.008 \\
\hline $\mathrm{uIgG} / \mathrm{uCr}(\mathrm{mg} / \mathrm{g})$ & $174.40(6.95-3373.12)$ & $705.53(38.86-11544.30)$ & 0.025 \\
\hline $\mathrm{uCRP} / \mathrm{uCr}(\mathrm{mg} / \mathrm{g})$ & 0.014 (BDL-1.07) & 0.47 (BDL-17.63) & 0.001 \\
\hline & $\operatorname{BDL}(n=1) ; B Q L(n=2)$ & BDL $(n=1)$ & \\
\hline uRBP/uCr (mg/g) & $0.83(0.29-2.07)$ & $0.92(0.26-7.71)$ & 0.51 \\
\hline uNGAL/uCr $(\mu \mathrm{g} / \mathrm{g})$ & $322.32(43.01-602.81)$ & $401.38(77.44-2645.43)$ & 0.11 \\
\hline pNGAL (ng/mL) & $13.08(7.46-31.25)$ & $10.50(5.95-121.77)$ & 0.73 \\
\hline $\mathrm{u} / \mathrm{pNGAL}$ & $55.23(21.52-110.00)$ & 50.99 (19.98-208.54) & 0.90 \\
\hline $\operatorname{sSDMA}(\mu \mathrm{g} / \mathrm{dL})$ & $13(8-23)$ & $17(10-45)$ & 0.038 \\
\hline
\end{tabular}

665 sCr: serum creatinine; USG: urine specific gravity; uOsmol: urine osmolality; UPC: urinary protein to creatinine ratio; uIgG: urinary

666 immunoglobulin G; uCr: urinary creatinine; uCRP: urinary C-reactive protein; BDL: below detection limit; BQL: below quantification limit;

667 uRBP: urinary retinol-binding protein; uNGAL: urinary neutrophil gelatinase-associated lipocalin; pNGAL: plasma neutrophil gelatinase-

668 associated lipocalin; u/pNGAL: urinary to plasma neutrophil gelatinase-associated lipocalin ratio; sSDMA: serum symmetric

669 dimethylarginine.

670

671

672

673

674

675

676

677

678

679 
680 Fig. 1. Kidney injury biomarkers (A: uIgG/uCr; B: uCRP/uCr; C: uRBP/uCr; D: uNGAL/uCr; E: pNGAL; F: u/pNGAL) and serum SDMA (G)

681 in healthy control dogs $(H, n=10)$ and dogs with babesiosis at presentation $\left(B T_{0}, n=35\right), 24$ hours after presentation $\left(B T_{24 h}, n=11\right)$, and 1

682 month after treatment $\left(\mathrm{B}_{1 \mathrm{~m}}, \mathrm{n}=9\right)$. Data are presented as boxes and whiskers. Each box includes the interquartile range and the line within

683 each box represents the median. The whiskers represent the range, extending to a maximum of 1.5 times the interquartile range. Outliers

684 are not shown. *Significantly different $(\mathrm{P}<0.05)$ from healthy control dogs. ${ }^{* *}$ Significantly different $(\mathrm{P}<0.05)$ from dogs with babesiosis at 685 presentation. 

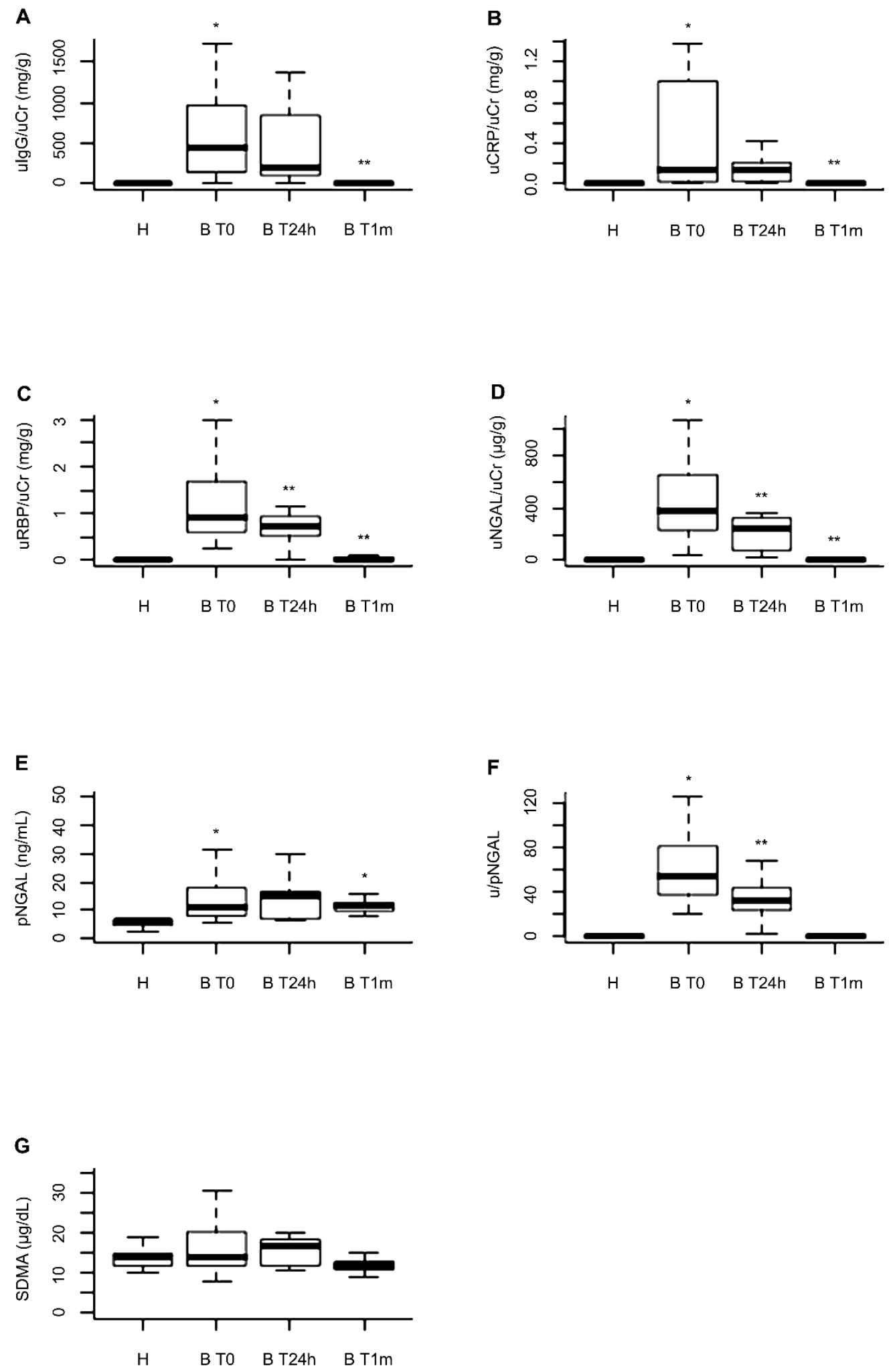